\section{T CELL PHENOTYPE DRIVES RESTRUCTURING OF TUMOR MICROENVIRONMENT TO BALANCE T CELL LONGEVITY AND TUMOR CONTROL: INSIGHTS FROM MULTIPLEXED IMAGING AND MULTI-SCALE AGENT BASED MODELING}

John Hickey*, Garry Nolan, Markus Covert, Eran Agmon, Nina Horowitz, John Sunwoo. Stanford University, Stanford, CA, USA

Background Immune cell therapies continue to have success in treatment of cancers yet face challenges of complexity, cost, toxicity, and low solid-tumor efficacy. Much work has focused on the phenotype characterization and control of ex vivo expanded cells; however, little is known about its relationship to changes in the tumor microenvironment in vivo. Thus, we imaged tumors treated with different phenotype tumor-specific $\mathrm{CD} 8+\mathrm{T}$ cells with CODEX multiplexed imaging ${ }^{1-4}$ that is able to visualize 42 antibodies at the same tissue in the tissue (figure 1A). To further probe this data in a systems immunology approach we created a multiscale agent-based model including critical circuits from the $\mathrm{T}$ cell-tumor microenvironment interactions (figure 1B).

Methods We initialized our agent-based models various percentages of either PD1+, PD1-, PDL1+, or PDL1- phenotypes and ran simulations for 72 hours. We also treated PMEL CD8 $+\mathrm{T}$ cells with or without 2 hydroxycitrate as a metabolic inhibitor during activation to achieve different input phenotypes of CD8 $+\mathrm{T}$ cells for therapeutic adoptive transfer on day 10 following B16-F10 tumors had been established. We performed neighborhood analysis on CODEX multiplexed imaging data by clustering neighboring cell types using a sliding window for neighborhood analysis.

Results Interestingly, the agent-based modeling indicated that the tumor phenotype switch to decrease proliferation was more effective than direct $\mathrm{T}$ cell killing. We observed spatially restricted inflammatory immune fronts when simulating with different initial percentages of PD1 $+\mathrm{T}$ cells and also from our CODEX multiplexed imaging. Quantitatively we observe that there is a drastic increase in the PDL1+, MHCI+, Ki67tumor phenotype that increases with metabolically inhibited $\mathrm{T}$ cells. Neighborhood analysis indicated that metabolically treated $\mathrm{T}$ cells were able to create distinct immune cell environments that supported productive $\mathrm{T}$ cell-tumor interactions and also helped maintain $\mathrm{T}$ cell phenotype.

Conclusions This indicates there is a balance for therapeutic $\mathrm{T}$ cell to mitigate chronic tumor exposure while controlling tumor growth through killing and by changing tumor phenotype. We observe $\mathrm{T}$ cells create distinct tumor microenvironments that differs significantly based on the starting $\mathrm{T}$ cell phenotype. Controlling $\mathrm{T}$ cell phenotype to promote productive immune-tumor structures will be critical to maintain $\mathrm{T}$ cell functionality and efficacy. In the future we will investigate $\mathrm{T}$ cell recruitment of immune structures by similar systems biology technologies.

Acknowledgements J.W.H. is funded by an ACS Postdoctoral Fellowship (PF-20-032-01-CSM).

\section{REFERENCES}

1. Goltsev Y, Samusik N, Kennedy-Darling J, Bhate S, Hale M, Vazquez G, Black S and Nolan GP, Deep profiling of mouse splenic architecture with CODEX multiplexed imaging. Cell, 174(4):968-981.

2. Schürch CM, Bhate SS, Barlow GL, Phillips DJ, Noti L, Zlobec I, Chu P, Black S, Demeter J, Mcllwain DR and Samusik N. Coordinated cellular neighborhoods orchestrate antitumoral immunity at the colorectal cancer invasive front. Cell 182 (5):1341-1359.
3. Black S, Phillips D, Hickey JW, Kennedy-Darling J, Venkataraaman VG, Samusik N, Goltsev Y, Schürch CM. and Nolan GP. CODEX multiplexed tissue imaging with DNA-conjugated antibodies. Nature Protocols 1-36.

4. Kennedy-Darling J, Bhate SS, Hickey JW, Black S, Barlow GL, Vazquez G, Venkataraaman VG, Samusik N, Goltsev Y, Schürch CM and Nolan GP. Highly multiplexed tissue imaging using repeated oligonucleotide exchange reaction. European Journal of Immunology 51(5):1262-1277.

Ethics Approval All studies involving mice were approved under Stanford's APLAC protocol 33502.

http://dx.doi.org/10.1136/jitc-2021-SITC2021.180 